\title{
Improved Understanding of Dense Jet Dynamics to Guide Management of Desalination Outfalls
}

\author{
M.J. Baum $^{\text {a }}$, B. Gibbes ${ }^{\text {a }}$ \\ a Aquatic Systems Research Group, School of Civil Engineering, The University of Queensland, Queensland, \\ Australia \\ Email: mitchell.baum@uqconnect.edu.au
}

\begin{abstract}
With the growing adoption of Seawater Reverse Osmosis desalination technologies, there is a concurrent increase in the production of dense, hypersaline by-products. To minimize environmental impact to benthic biota, these wastes are commonly disposed via submerged diffuser systems in dynamic coastal environments. Traditional diffuser design approaches have relied on empirical techniques derived from smallscale laboratory experiments. This approach has provided a sound basis for preliminary design and regulatory approval of these systems. In practice the coastal receiving environment differs from the idealistic laboratory environments from which empirical scaling functions were derived. With the recent advances in computational power and development of computational fluid dynamics (CFD) approaches, it is now feasible to utilize CFDbased analysis to examine the dynamics of dense brine plumes under conditions representative of in-situ field practices.
\end{abstract}

For the first time, this study details a high-resolution three-dimensional laboratory-scale numerical simulation of an inclined dense jet diffuser subject to ambient crossflow. The quasi-steady CFD simulations were performed using the Reynolds averaged Navier-Stokes equations with a $k$ - $\omega$ shear stress transport turbulence closure scheme. The study compliments existing laboratory studies by assessing CFD simulation results against empirical scaling approaches. Quantitative assessment of diffuser performance with regard to trajectory and dilution for an array of dynamic-crossflow based regimes is presented.

Results show strong agreement with existing small-scale laboratory experiments, with significant potential for upscaling to field-scale applications. Simulated dynamic ambient regimes show the influence of crossflow upon jet trajectory, dilution and lower boundary concentration is significant. The effect of flow structure and the subsequent influence on jet dynamics is discussed. This model poses as an effective strategy for future design of brine outfall systems, with strong potential for application in water quality management for both plant operators and regulators.

Keywords: Inclined dense jets, desalination, computational fluid dynamics, OpenFOAM 
Baum and Gibbes, Improved Understanding of Dense Jet Dynamics to Guide Management of Desalination Outfalls

\section{INTRODUCTION}

With the escalating global need to secure climate resilient water supply, seawater desalination has become increasingly adopted in recent decades (Villacorte et al., 2015). This desalting process is most commonly undertaken using Seawater Reverse Osmosis (SWRO) technologies. Hypersaline brines are a by-product of this process and are typically discharged into oceanic or estuarine systems. Due to the elevated salinity content and negligible thermal inputs during the recovery process, SWRO reject streams are characteristically denser than their receiving environment. To minimize impacts on benthic marine biota, SWRO brines are disposed via submerged, inclined seabed diffuser systems to enhance mixing and dilution of the plume and thus reducing potential ecological impacts. A jet inclination of $60^{\circ}$ above horizontal is widely used for these discharges based on the outcome of past laboratory-scale experiments (e.g. Zeitoun et al., 1970; Abessi and Roberts, 2015).

The hydrodynamics of inclined dense jets are influenced by the interplay of momentum and buoyancy. Attributes of these flows are conceptualized in Figure 1. Effluent with density, $\rho_{0}\left[\begin{array}{ll}\mathrm{M}^{-3} & \mathrm{~L}^{-3}\end{array}\right.$ is discharged with velocity, $U_{0}\left[\mathrm{~L} \mathrm{~T}^{-2}\right]$ via a sharp-edged circular orifice with diameter, $d[\mathrm{~L}]$, into a receiving water body with density, $\rho_{a}\left[\mathrm{M} \mathrm{L}^{-3}\right]$. With the high discharge velocity, the flow is initially dictated by jet momentum. However, due to the elevated density of the brine reject stream $\left(\rho_{0}>\rho_{a}\right)$, buoyancy forces cause the jet to reach a terminal height $\left(Z_{t}\right)$, before descending to

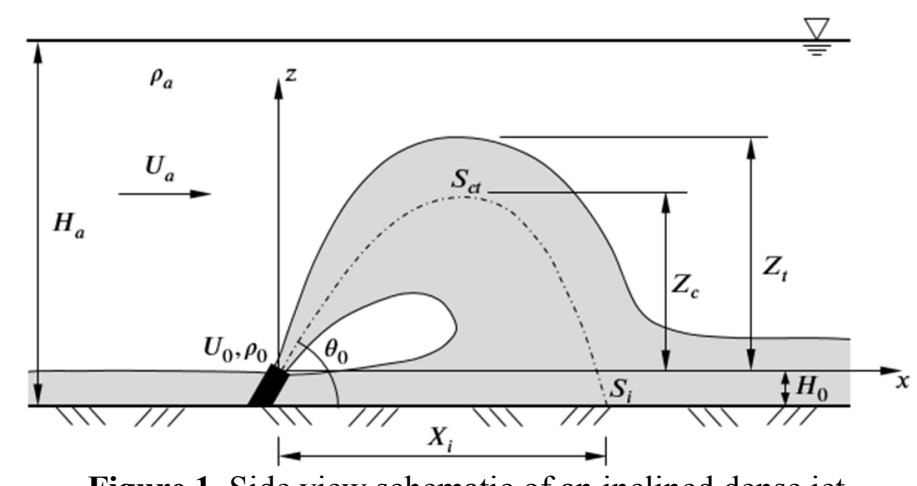

Figure 1. Side view schematic of an inclined dense jet illustrating key jet characteristics. the lower seafloor boundary and spreading radially as a density current (Roberts et al., 1997).

Several experimental studies have examined the behavior of inclined dense jets subject to ambient crossflow. Assuming fully turbulent flow and validity of the Boussinesq approximation applies (i.e. $\left.\left(\rho_{0}-\rho_{a}\right) \ll \rho_{a}\right)$, several studies (Tong and Stolzenbach, 1979; Roberts and Toms, 1987; Lai and Lee, 2014; Abessi and Roberts, 2017) have identified that for a singular discharge port with a fixed inclination angle subject to co-propagating current, trajectory $(\chi)$ and dilution $(S)$ parameters are given as:

$$
\frac{\chi}{d F}, \frac{S}{F}=f\left(u_{r} F\right)
$$

where $F[-]$ is the jet densimetric Froude number (i.e. $\left.F=U_{0} /\left(g_{0}^{\prime} \times d\right)^{1 / 2}\right)$ and $g_{\boldsymbol{0}}=g \times\left(\rho_{0}-\rho_{a}\right) / \rho_{a}\left[\mathrm{~L} \mathrm{~T}^{-2}\right]$ is the modified acceleration due to gravity. The term $u_{r} F[-]$, is a form of ambient crossflow Froude number, which relates the magnitude of ambient current, $U_{a}\left[\mathrm{~L} \mathrm{~T}^{-1}\right]$, to jet exit velocity with the ratio $u_{r}=U_{a} / U_{0}[-]$. For $u_{r} F \ll 1$, flow parameters are dictated by jet momentum and buoyancy properties, while for $u_{r} F>\sim 0.5$ the effects of ambient crossflow are deemed significant (Roberts, 2015).

Traditional design approaches employ empirical scaling arguments that were developed from small-scale laboratory studies or integral entrainment methods. While these approaches have provided a sound basis for design and regulation of brine diffuser systems, their ability to accommodate complex port geometries, stratification of the receiving water body and ambient hydrodynamic processes consistent with field-scale dense outfall applications is limited. With the increase of computational power and advancement of computational fluid dynamics (CFD) tools, several studies (Oliver, 2008; Seil and Zhang, 2010; Gildeh et al., 2015; Zhang et al., 2017) have used numerical methods to examine near-field behavior of inclined dense jets in quiescent ambient environments. While these studies have demonstrated significant advancements over commercial integral entrainment models, no existing numerical CFD studies have examined inclined dense jets subject to ambient crossflow.

In this study, a quasi-steady numerical model using a Reynolds averaged approach to resolve the Navier-Stokes equations was used to simulate a $60^{\circ}$ inclined dense jet subject to several co-propagating regimes. The objective of this research was to evaluate the performance of the near-field model projections against existing laboratorybased research. Details of the computational methodology are provided along with a quantitative assessment of key trajectory and dilution outcomes. 
Baum and Gibbes, Improved Understanding of Dense Jet Dynamics to Guide Management of Desalination Outfalls

\section{MATERIALS AND METHODS}

Simulations were conducted using the open-source CFD utility, OpenFOAM (OpenFOAM, 2013). Specifically, the incompressible multiphase solver employed a variant of the twoLiquidMixingFoam solver to resolve quasi-steady solutions for the Reynolds Averaged Navier Stokes (RANS) equations using the Finite Volume Method (FVM). The twoLiquidMixingFoam solver has been used and validated in many studies (Lai et al., 2015; Zhang et al. 2017). A $k$ - $\omega$ Shear Stress Transport (SST) turbulence closure scheme for the RANS equations was utilized. Consistent with Zhang et al. (2017) simulations used a turbulent Schmidt number of 0.7 . A diffusivity of $1 \times 10^{-8} \mathrm{~m}^{2} \mathrm{~s}^{-1}$ and fluid viscosity of $1 \times 10^{-6} \mathrm{~kg} \mathrm{~m}^{-1} \mathrm{~s}^{-1}$ were also used.

The model geometry is illustrated in Figure 2(A) and was designed to replicate the laboratory setup of Roberts et al. (1997). The model domain was $0.91 \mathrm{~m}$ wide and $0.61 \mathrm{~m}$ deep, while a reduced longitudinal length of 2.40 $\mathrm{m}$ was used. The simulated diffuser port has a diameter, $d=4.29 \times 10^{-3} \mathrm{~m}$, with a centroid elevation of $25 \times 10^{-3}$ $\mathrm{m}$ above the bottom of the domain which was simulated as a smooth boundary. The model domain was discretized using a hexahedral mesh with a maximum cell dimension of $15 \times 10^{-3} \mathrm{~m}$. An adaptive mesh utility was used to resolve jet hydrodynamics in the near-field. This was iteratively implemented on the basis of a threshold concentration value for which a cell with a local discharge concentration (c) exceeding $3 \%$ of the initial discharge concentration, $C_{0}$, (i.e. $c / C_{0}>0.03$ ) was successively refined. After 3,000 iterations the model cell count subsequently increased from $\sim 400,000$ cells to over $1,100,000$ cells.

(A)

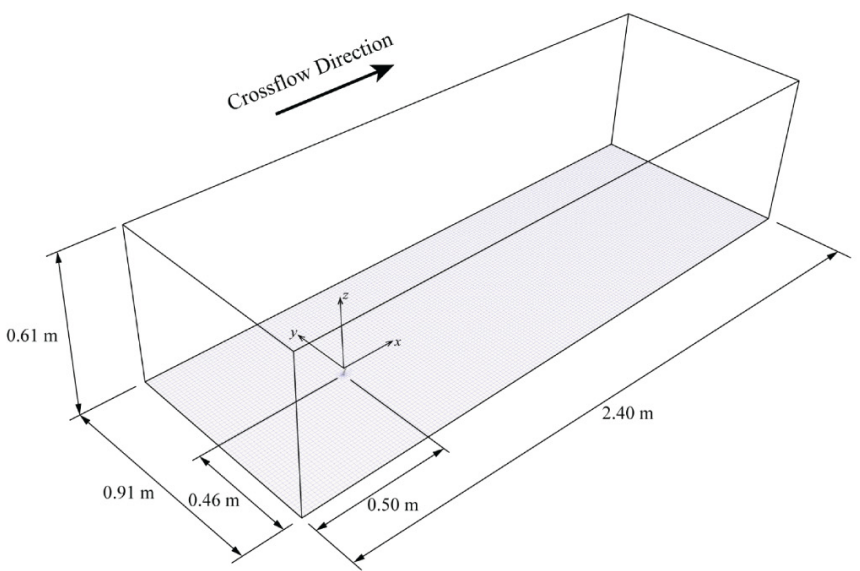

(B)

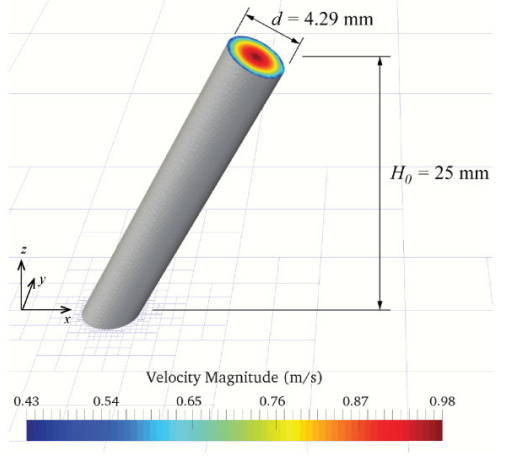

Figure 2. (A) Projection of model domain and general model dimensions. (B) Nozzle exit velocity boundary condition and dimensions.

Discharge and ambient densities were $\rho_{0}=1030 \mathrm{~kg} \mathrm{~m}^{-3}$ and $\rho_{a}=997 \mathrm{~kg} \mathrm{~m}^{-3}$, respectively. The discharge velocity boundary condition was accommodated assuming fully developed turbulent pipe flow. Constituting a discharge Froude number of $F=20$, a mean velocity across the nozzle inlet of $\overline{U_{0}}=0.75 \mathrm{~m} \mathrm{~s}^{-1}$ was used to define the power-law discharge velocity boundary condition (Figure 2(B)) with a Reynolds number of $R e=$ 3200. In accordance with Stigler (2014) the mean velocity profile as a function of the nozzle radial distance, $r$ [L], is given by:

$$
\bar{u}(r)=\frac{\overline{U_{0}}}{2}\left(\frac{1}{n}+1\right)\left(\frac{1}{n}+2\right)\left(1-\frac{r}{\frac{d}{2}}\right)^{\frac{1}{n}}
$$

where $n$ is an empirical constant given by $n=1.03 \ln (\operatorname{Re})-3.6[-]$.

A slip boundary condition was imposed at the top surface, while a Dirichlet condition was set at the model outlet. Neumann boundary conditions were set at all other boundary interfaces, with zero velocity conditions imposed at the floor and port boundaries.

Initial approximations for turbulent kinetic energy, $k\left[\mathrm{~L}^{2} \mathrm{~T}^{-2}\right]$, and specific dissipation rate, $\omega\left[\mathrm{T}^{-1}\right]$, were determined using:

$$
k=\frac{3}{2}(\bar{U} I)^{2}
$$


Baum and Gibbes, Improved Understanding of Dense Jet Dynamics to Guide Management of Desalination Outfalls

$$
\omega=\frac{k^{1 / 2}}{C_{\mu}^{1 / 4} \ell}
$$

where the coefficient of viscosity, $C_{\mu}=0.09[-]$, is an empirical constant. The turbulence length scale was approximated as $\ell=0.07 L[\mathrm{~L}]$, where $L[\mathrm{~L}]$ is the hydraulic diameter. The nozzle turbulent intensity, $I[-]$ was approximated by $I_{0}=0.16 R e^{-1 / 8}=5.8 \%$, while an ambient turbulent intensity of $I_{\text {ambient }}=5.0 \%$ was defined.

\section{RESULTS AND DISCUSSION}

Five simulations were conducted in this investigation with discharge and ambient transport properties held constant across each case, while ambient crossflow scenarios distinguish the different regimes (Table 1). Crossflow magnitudes ranging $u_{r} F=0.0-2.0$ were examined, with ambient velocities reaching up to $10.1 \%$ of mean jet exit velocity $\left(\overline{U_{0}}\right)$. At prototype scale with $d=240 \mathrm{~mm}, F=20$ and equivalent densimetric properties, these crossflow conditions equate to ambient velocities ranging $U_{a}=0.00-0.61 \mathrm{~m} \mathrm{~s}^{-1}$. To ensure quasi-steady simulation and attain adequate mesh refinement to resolve the jet trajectory, simulations were carried out for 3,000 iterations. This value was determined by examining the convergence of jet trajectory and concentration.

Table 1. Summary of numerical simulation parameters.

\begin{tabular}{|c|c|c|c|c|c|c|c|}
\hline $\begin{array}{c}\text { Case } \\
\text { ID }\end{array}$ & $F(-)$ & $U_{a}\left(\mathrm{~m} \mathrm{~s}^{-1}\right)$ & $\overline{U_{0}}\left(\mathrm{~m} \mathrm{~s}^{-1}\right)$ & $u_{r}(\%)$ & $u_{r} F(-)$ & $\begin{array}{l}\text { No. Cells } \\
\text { (Millions) }\end{array}$ & $\begin{array}{c}\text { Simulation } \\
\text { Time (Hours) }\end{array}$ \\
\hline $\begin{array}{l}\text { QU1 } \\
\end{array}$ & 20 & 0.000 & 0.746 & 0.0 & 0.0 & 1.18 & 6.9 \\
\hline $\mathrm{CO} 1$ & 20 & 0.019 & 0.746 & 2.5 & 0.5 & 1.22 & 9.7 \\
\hline $\mathrm{CO} 3$ & 20 & 0.056 & 0.746 & 7.5 & 1.5 & 1.59 & 10.1 \\
\hline $\mathrm{CO} 4$ & 20 & 0.075 & 0.746 & 10.1 & 2.0 & 1.62 & 10.5 \\
\hline
\end{tabular}

\subsection{General Observations}

Normalized brine concentration distributions for each co-propagating crossflow discharge scenario are presented in Figure 3. Unlike the widely applied integral entrainment models that assume self-similar vertical Gaussian concentration distributions along the length of the jet trajectory (Roberts, 2015), the CFD method used here accounts for gravitationally induced detrainment on the internal region of the jet. Consistent with results presented by Abessi and Roberts (2017), this detrainment-induced asymmetry decreases with increasing crossflow magnitude. With the introduction of crossflow consistent trends of increasing trajectory elongation and dilution properties are observed.
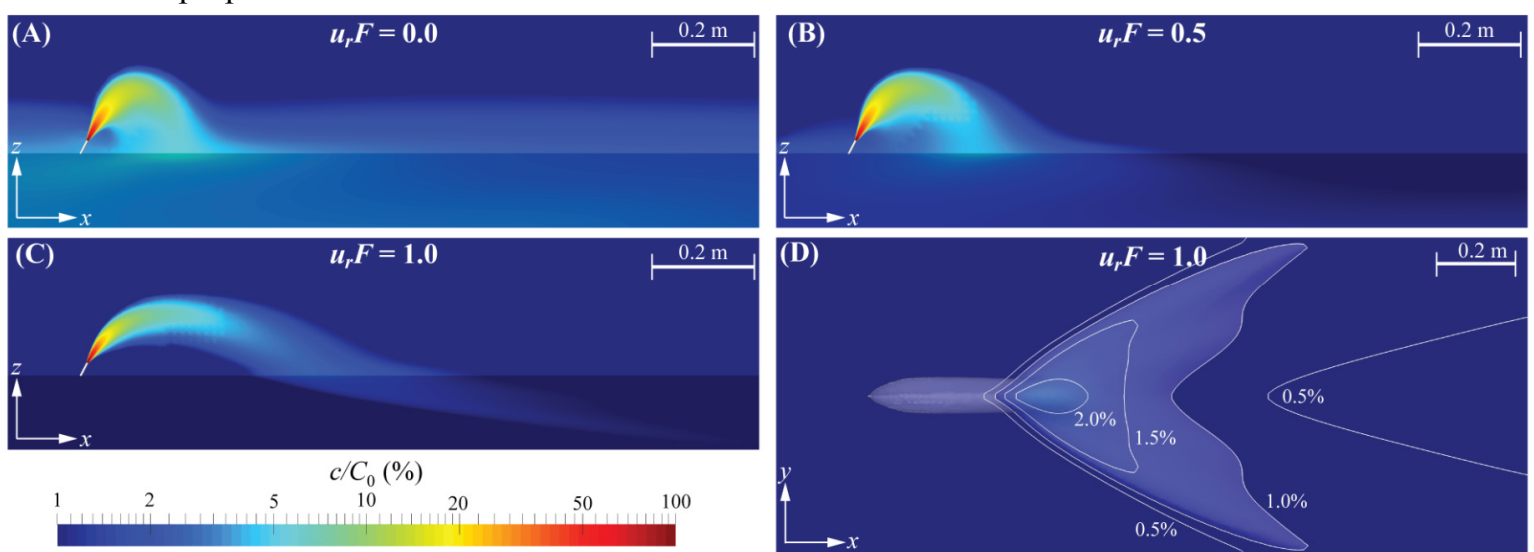

Figure 3. Normalized concentration distributions. Current propagates from left to right. Logarithmic color scale used. (A) - (C) show three-dimensional transects taken through the central plane and lower boundary concentration. (D) shows a plan view of the jet with the outer transparent bound of the discharge denoted by a concentration of $c / C_{0}=1 \%$. Concentration contours along the lower boundary are shown.

For the quiescent discharge case $\left(u_{r} F=0.0\right)$ the dense sublayer envelopes most of the trajectory, with only the vertical terminus of the jet exceeding this residual layer. The presence of the dense sublayer appears to be highly sensitive to the presence of crossflow. No residual layer is evident beyond the point of impact for $u_{r} F>$ 0.0. Consistent with Choi et al. (2016) an arrested upstream wedge egression occurs for $u_{r} F=0.5$ (Figure 3(B)) - albeit propagating slightly further upstream than observed by Choi et al. (2016) where for $u_{r} F=0.56$ the wedge was located slightly downstream of the discharge point. Additional agreement with the results of Choi et al. (2016) is found for $u_{r} F>0.8$ where no upstream wedge is present. With the absence of the upstream 
Baum and Gibbes, Improved Understanding of Dense Jet Dynamics to Guide Management of Desalination Outfalls

wedge and density-induced sublayer for $u_{r} F \geq 1.0$ re-entrainment of discharge is avoided, thus facilitating further entrainment of fresh ambient fluid. Consistent with Gungor and Roberts (2009), Choi et al. (2016) and Meftah et al. (2017) the transition from radial dissipation to bifurcated jet flow (Figure 3(D)) after impacting the lower boundary is observed in the intermediate field for $u_{r} F \geq 1.0$. Meftah et al (2017) shows this flow behavior arises due to the presence of counter-rotating vortices induced after impact.

\subsection{Impact Distance}

The impact distance of the jet $\left(X_{i}\right)$ is the horizontal distance from the port to the location where the concentration centerline impacts the lower boundary. The non-dimensional impact distance for each simulated regime is shown in Figure 4(A). Modelled impact distances show strong correlation with existing laboratorybased crossflow studies, exhibiting an exponential trend of increasing impact distance with increasing crossflow magnitude. Comparison of the quiescent scenario against the experimental study of Roberts et al. (1997) and the more recent LES model of Zhang et al. (2017) shows this present model is slightly conservative in regards to impact distance. This could be taken as evidence that the quasi-steady RANS based approach is limited in its ability to resolve transport dictated by the transient advection processes that govern the entrainment upon descent of jet-dictated discharges. For the dynamic ambient regimes, flow behavior becomes dictated by crossflow and modelled outcomes fall within the spread of existing laboratory studies.
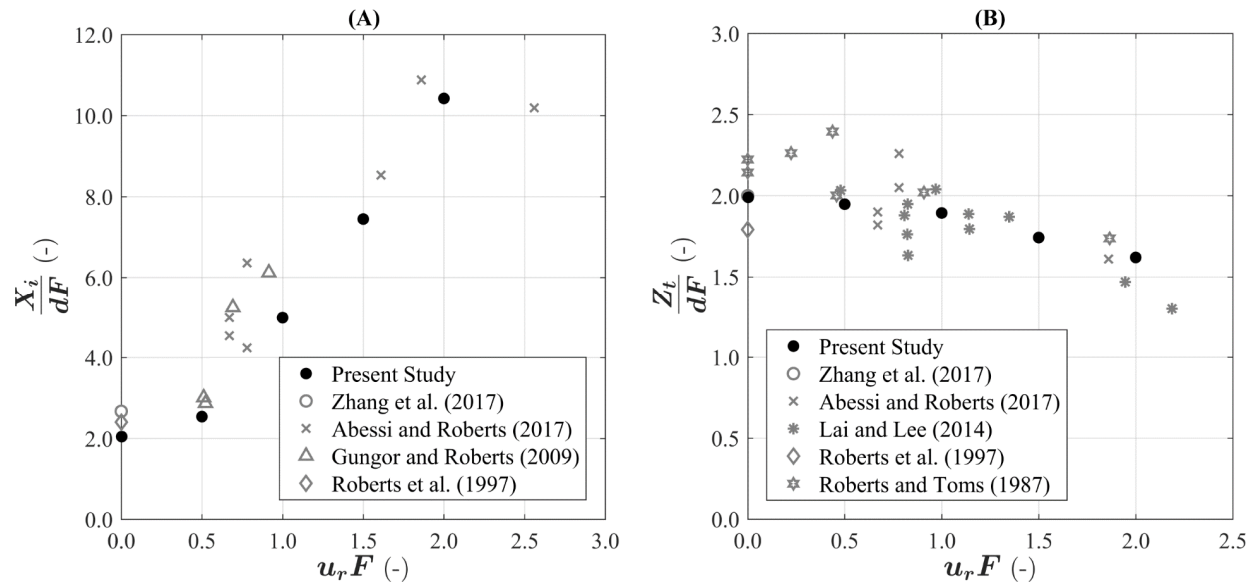

Figure 4. Non-dimensional jet trajectory properties with crossflow: (A) impact distance, $X_{i}$, and (B) terminal boundary elevation, $Z_{t}$. Terminal rise heights obtained from Roberts et al. (1997) and Roberts and Toms

(1987) measured relative to bottom boundary and adjusted to account for port discharge elevation.

\subsection{Terminal Rise}

The terminal rise height $\left(Z_{t}\right)$ is the elevation above the discharge source to the outer extremity of the jet. This distance is determined as the maximum elevation where the local concentration $(c)$ is $10 \%$ of the jet centerline value at its vertical terminus. The non-dimensional terminal rise for each simulation is presented in Figure 4(B). For the quiescent scenario this boundary concentration was $c=1.16 \%$ while for $u_{r} F=2.0$, the boundary concentration was $c=0.47 \%$. Simulated terminal extremities show overall trends of decreasing terminal extremity with increasing crossflow magnitude. Comparison with jet-dominated flows $\left(u_{r} F \leq 0.5\right)$ shows the model is conservative when compared against data from Roberts and Toms (1987). This may again arise from the quasi-steady RANS simulation approach whereby the advective 'puffs' that occupy the terminal extremity of the jet (Abessi and Roberts, 2014) are not directly accommodated. Despite this, the quiescent model concurs with the time-averaged LES models of Zhang et al. (2017). With increasing $u_{r} F$, these 'puffs' are increasingly dictated by crossflow and subsequently their propagation over the vertical extent of the water column is reduced. This is reflected by the increasing degree of confidence observed for crossflow dictated regimes, with the model adhering to the spread of experimental data over the simulated crossflow range.

\subsection{Centerline Terminal Rise Dilution}

The centerline terminal rise dilution, $S_{c t}$, is defined as the minimum dilution recorded at the terminal elevation of the jet concentration centerline. The terminal rise dilutions from the quasi-steady RANS predictions are shown in Figure 5(A) and demonstrate consistent trends of increasing dilution with increasing crossflow magnitude. Predictions are in good agreement with Lai and Lee (2014). For $F>20$, a direct fit from data extrapolated from Lai and Lee (2014) shows $S_{c t}=0.71 u_{r} F^{1 / 2}$ while this study yields $0.76 u_{r} F^{1 / 2}$. However, this validation should be considered with discretion as Lai and Lee (2014) examined jet discharging perpendicular 
Baum and Gibbes, Improved Understanding of Dense Jet Dynamics to Guide Management of Desalination Outfalls

to crossflow. Conversely, Roberts and Toms (1987) identified a $u_{r} F^{1 / 3}$ trend for co-propagating crossflow using a crude vacuum sampling method. Jet-dictated scenarios $\left(u_{r} F<1.0\right)$ are in general agreeance with Roberts and Toms (1987), Lai and Lee (2012) and Choi et al. (2016); while dilution slightly exceeds the LES prediction of Zhang et al. (2017) under quiescent conditions.
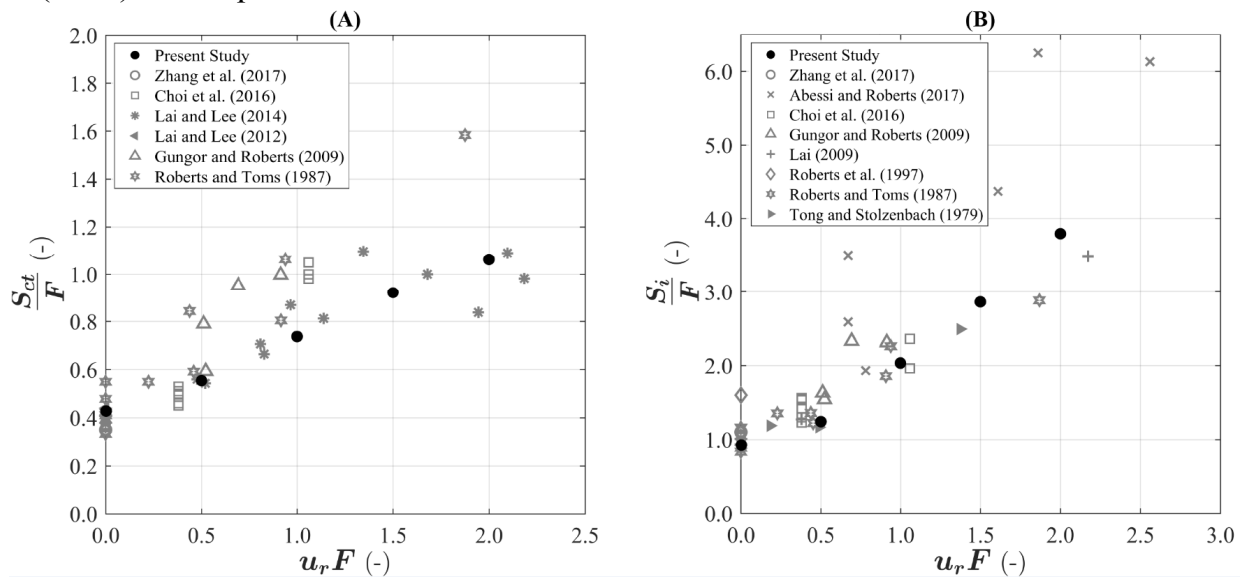

Figure 5. (A) Minimum dilution at the location of terminal elevation of the concentration centerline. (B)

Dilution at the jet impact distance.

\subsection{Impact Dilution}

The impact dilution is the minimum dilution at the return of the jet trajectory to the lower boundary. This parameter is of key interest to the design, regulation and operation of these outfalls with regard to impacts to benthic marine communities. Simulated impact dilutions are presented in Figure 5(B). For $u_{r} F=0.0$, impact dilution appears conservative relative to Roberts et al. (1997), although the model is in general agreeance with equivalent data extrapolated from Roberts and Toms (1987). Quiescent LES simulations from Zhang et al. (2017) achieved a slightly higher dilution upon impact with $S_{i} / F=1.10$ compared to $S_{i} / F=0.93$ obtained in this study. Note that these are significant improvements to existing commercial integral entrainment models where Palomar et al. (2012) determined $S_{i} / F=0.62,0.70$ and 0.79 for UM3, JetLag and CORJET respectively.

Similar to the impact distance, impact dilution exhibits an exponential trend of increasing dilution with increasing crossflow magnitude and shows good agreement with similar studies concerning singular discharge ports subject to crossflow (Choi et al., 2016; Gungor and Roberts, 2009; Lai, 2009; Roberts and Toms, 1987; and Tong and Stolzenbach, 1979).

\section{CONCLUSION}

A laboratory-scale numerical investigation of a singular dense jet with an inclination of $\theta_{0}=60^{\circ}$ was conducted for various co-propagating crossflow conditions. The simulations used a quasi-steady RANS approach with a $k-\omega$ SST closure scheme to resolve the flow, whereby trajectory and dilution parameters were evaluated quantitatively. This numerical study showed strong correlation with existing laboratory measurements of inclined dense jets in crossflow environments. Results demonstrated the potential of CFD as an effective predictive tool to design and manage brine outfalls. Based on numerical results and comparisons with existing modelling studies and laboratory investigations, the following conclusions can be made:

- Under quiescent receiving conditions, the model provides significant improvements over existing integral entrainment modelling tools including CORMIX, UM3 and JetLag in terms of terminal elevation and dilution parameters.

- While empirical methods are still superior under quiescent conditions, the numerical model presented here provides a very promising tool to assess dilution and trajectory properties of these outfalls in dynamic ambient environments. CFD methodologies have potential to facilitate the analysis of discharges in situations where empirical models are unable to account for variations such as complex port geometries, ambient mechanisms (e.g. stratification, velocity shear etc.), seafloor slope and irregular substrate geometry.

- While the LES numerical model of Zhang et al. (2017) yielded slightly improved predictions of both impact distance and impact dilution, the quasi-steady RANS approach presented here is much more computationally efficient. For 180 seconds of simulated flow on up to 128 cores, real-time computing for the LES model required up to 20 days. In contrast, the equivalent quiescent regime presented here was solved across 16 cores in $\sim 7$ hours. 
Baum and Gibbes, Improved Understanding of Dense Jet Dynamics to Guide Management of Desalination Outfalls

Further numerical studies may wish to consider the development of a turbulent closure scheme to specifically resolve mixing and plume behavior inherent with the gravitational instabilities that govern the mixing of the jet. More broadly, numerical methods may further be applied to consider counter-propagating crossflow regimes and multiport diffuser behavior.

\section{ACKNOWLEDGMENTS}

The authors acknowledge the financial support of the National Centre of Excellence in Desalination Australia, which is funded by the Australia Government through the Water for the Future Initiative. The authors also acknowledge Greg Collecutt who contributed to the underlying model utilities used in this study.

\section{REFERENCES}

Abessi, O., and Roberts, P. J. W. (2015). Effect of Nozzle Orientation on Dense Jets in Stagnant Environments. Journal of Hydraulic Engineering, 141(8), 06015009.

Abessi, O., and Roberts, P. J. W. (2017). Multiport Diffusers for Dense Discharge in Flowing Ambient Water. Journal of Hydraulic Engineering. 143(6), 04017003.

Choi, K. W., Lai, C. C. K., and Lee, J. H. W. (2016). Mixing in the Intermediate Field of Dense Jets in Cross Currents. Journal of Hydraulic Engineering, 142(1), 04015041.

Gungor, E., and Roberts, P. J. W. (2009). Experimental Studies on Vertical Dense Jets in a Flowing Current. Journal of Hydraulic Engineering, 135(11), 935-948.

Gildeh, H. K., Mohammadian, A., Nistor, I., Qiblawey, H., and Yan, X. (2015). CFD modeling and analysis of the behavior of $30^{\circ}$ and $45^{\circ}$ inclined dense jets - new numerical insights. Journal of Applied Water Engineering and Research, 2(4), 1-16.

Lai, C. C. K. (2009). Mixing of inclined dense jets (M.Phil thesis, University of Hong Kong, Hong Kong).

Lai, C. C. K., and Lee, J. H. W. (2012). Mixing of inclined dense jets in stationary ambient. Journal of Hydroenvironment Research, 6(1), 9-28.

Lai, C. C. K., and Lee, J. H. W. (2014). Initial mixing of inclined dense jet in perpendicular crossflow. Environmental Fluid Mechanics, 14(1), 25-49.

Lai, A. C. H., Zhao, B., Law, A. W. K., and Adams, E. E. (2015). A numerical and analytical study of the effect of aspect ratio on the behavior of a round thermal. Environmental Fluid Mechanics, 15(1), 85-108.

Meftah, M. B., Malcangio, D., De Serio, F., and Mossa, M. (2017). Vertical dense jet in flowing current. Environmental Fluid Mechanics, 1-22.

Oliver, C. J., Davidson, M. J., and Nokes, R. I. (2008). k- $\varepsilon$ Predictions of the initial mixing of desalination discharges. Environmental Fluid Mechanics, 8(5-6), 617-625.

The OpenFOAM Foundation, OpenFOAM (Version 2.2.2), OpenCFD Ltd., 2013.

Palomar, P., Lara, J. L., and Losada, I. J. (2012). Near field brine discharge modeling part 2: Validation of commercial tools. Desalination, 290, 28-42.

Roberts, P. J. W., and Toms, G. (1987). Inclined Dense Jets in Flowing Current. Journal of Hydraulic Engineering, 113(3), 323-340.

Roberts, P. J. W., Ferrier, A., and Daviero, G. (1997). Mixing in Inclined Dense Jets. Journal of Hydraulic Engineering, 123(8), 693-699.

Roberts, P. J. W. (2015). Near Field Flow Dynamics of Concentrate Discharges and Diffuser Design. In T. M. Missimer, B. Jones, and R. G. Maliva (Ed.), Intakes and Outfalls for Seawater Reverse-Osmosis Desalination Facilities (369-396). Switzerland: Springer International Publishing.

Seil, G., and Zhang, Q. (2010). CFD modelling of desalination plant brine discharge systems. Water, 37(6), 79-83.

Stigler, J. (2014). Analytical velocity profile in tube for laminar and turbulent flow. Engineering Mechanics, 21(6), 371-379.

Tong, S. S., and Stolzenbach, K. D. (1979). Submerged Discharges of Dense Effluent, Report No. 243. Ralph M. Parsons Laboratory, Massachusetts Institute of Technology, Cambridge, Mass. Retrieved from: https://repository.tudelft.nl/islandora/object/uuid\%3A397fe811-d155-430c-83ad-735073f07dc6

Villacorte, L. O., Tabatabai, S. A. A., Dhakal, N., Amy, G., Schippers, J. C., and Kennedy, M. D. (2015). Algal blooms: an emerging threat to seawater reverse osmosis desalination. Desalination and Water Treatment, 55(10), 2601-2611.

Zeitoun, M. A., Reid, R. O., McHilhenny, W. F., and Mitchell, T. M. (1970). "Model studies of outfall system for desalination plants." Research and Development Progress Report. 804, Office of Saline Water, U.S. Dept. of Interior, Washington DC.

Zhang, S., Law, A. W.-K., \& Jiang, M. (2017). Large eddy simulations of $45^{\circ}$ and $60^{\circ}$ inclined dense jets with bottom impact. Journal of Hydro-environment Research, 15(1), 54-66. 